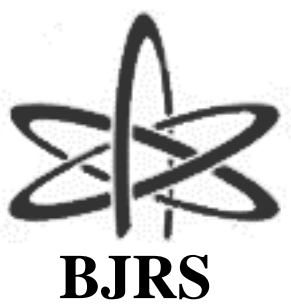

BRAZILIAN JOURNAL

$\mathrm{OF}$

RADIATION SCIENCES

06-01 (2018) 01-09

\title{
The image reconstruction influence in relative measurement in SPECT / CT animal
}

\author{
S.C.S. Soriano ${ }^{\text {a }}$ S.A.L. Souza ${ }^{\text {b }}$ T.Barboza ${ }^{\text {; }}$ L.V. De Sáa \\ ${ }^{a}$ Instituto de Radioproteção e Dosimetria (IRD)/Comissão Nacional de Energia Nuclear (CNEN), 22783-127 \\ Rio de Janeiro, $R J$ \\ sarahsoriano@bolsista.ird.gov.br \\ ${ }^{b}$ Hospital Universitário Clementino Fraga Filho(HUCFF)/Universidade Federal do Rio de Janeiro, 21941-913
}

Rio de Janeiro, $R J$

\begin{abstract}
The objective of this study was to evaluate the influence of different reconstruction methods in a FLEX Triumph TM Pre-Clinical Imaging System images applied to mice C57BL6 dosimetry in a new radiopharmaceuticals development for humans use. CT and SPECT images were obtained from a homemade phantom containing four spheres with diameters (d) simulating technetium-accumulating lesions at University Hospital (HU / UFRJ). The SPECT images were reconstructed with filtered back projection method (FBP) and the influence in resolution and partial volume effect was evaluated for different filters: Hamming, Hann and Ramp. The reconstructed image resolution was found 9.3 to $9.4 \mathrm{~mm}$, significantly lower than the value provided by the manufacturer, $1.6 \mathrm{~mm}$. Thus, the protocol for mice can be optimized by FBP reconstruction method and Hamming filter, 0.5 cut-off, yielding a reconstructed image resolution from 9.3 to $9.4 \mathrm{~mm}$. This value indicates that technetium-accumulating regions diameter below $9.3 \mathrm{~mm}$ is not quantifiable.
\end{abstract}

Keywords: SPECT/CT animal, reconstruction, preclinical study. 


\section{INTRODUCTION}

Animal models are used in new pharmacological compounds development related to human health since the 1950s, being a useful and efficient practice to avoid adverse effects (MarticKehl et al., 2012).

The image from SPECT (Single Photon Emission Computed Tomography) technique, also known as molecular imaging, is a non-invasive method to detect the signals originating from the injection of a radioactive tracer, observing the interaction of this with the target molecule in vivo (Meikle et al., 2005).

To guarantee diagnostic images quality for internal dosimetry purposes, the better reconstruction method analyses is necessary. This approach comprises a process of creating transaxial sections displaying a projection - filtered back projection (FBP) for the protocol applied in the studied system- where the raw data is used to create several transaxial slices. Figure 1 is a representation of this process; ten projection views are cut into seven separate bands. The corresponding bands form a radius, and so on, completing the image reconstruction process (Powsner, 2006).

Figure 1: Retroprojection example

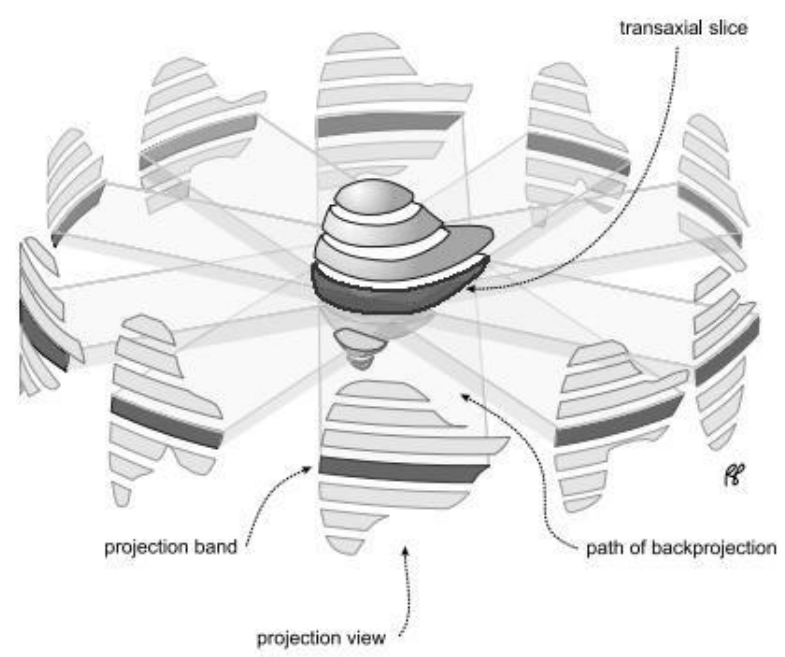

Source: Powsner, 2006. 
Subsequently, the images were filtered - a mathematical technique used during reconstruction to enhance the image "appearance". In particular, for diagnostic and dosimetric purposes, the filters are used to increase signal to noise ratio between distinct activity concentration areas in the image, to reduce "star" artefacts removing noise due to scattered photons and statistical variations in the count. The term cut-off is related to the maximum frequency that the filter could be applied, often referred to as the filter's "power". Low-pass filters (Butterworth, Hann, Hamming, Parzen) are used as pre-filters to remove high frequency noise. The Ramp filter, high-pass, is then applied during the backprojection for removing star and other low frequency data. The optimum filter selection depends on both the characteristics of the data and the personal preference of the user, influencing the final score obtained in the image and hence, the dosimetry. In general, the high-pass filter is more suitable for higher data counts, while the low-pass filter is best for data containing a small number of counts (Powsner, 2006).

\section{MATERIALS AND METHODS}

SPECT and CT (Computed Tomography) images were obtained from a homemade phantom (Figure 2) containing four spheres of different diameters (d), developed at the Institute of Radiation Protection and Dosimetry, based on the Micro Hollow Sphere Phantom ${ }^{\mathrm{TM}}$ Model ECT/HS/MMP (Data Spectrum Co.), as described in Table 1. These inserts simulate technetiumaccumulating lesions to be analysed by images acquired in FLEX Triumph TM Imaging System used in preclinical studies at the University Hospital (HU / UFRJ). The local SPECT protocol was: matrix $128 \times 128,20$ minutes acquisition total time, energy window $140 \mathrm{keV} \pm 15 \%$. CT protocol was: matrix 512_x_512, pitch $1.90 \mathrm{~mm}, 75 \mathrm{KV}$, FBP reconstruction method.

To simulate a real animal study, the total volume of the phantom (body) was filled with Technetium-99m solution diluted in water at an activity concentration of $0.629 \mathrm{MBq} / \mathrm{ml}$. The spheres were filled with a concentration four times higher compared to the body of the phantom. 
Soriano, et. Al. • Braz. J. Rad. Sci. • 2018

Figure 2: Phantom used in this study

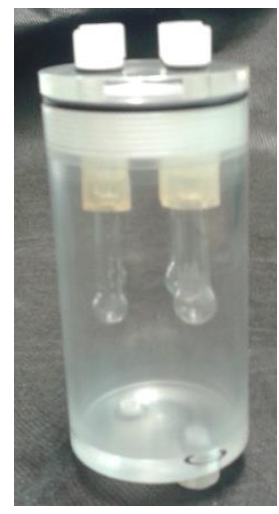

Table 1: Phantom description.

\begin{tabular}{ccccc}
\hline Sphere diameter (mm) & 4.8 & 7.1 & 9.0 & 9.6 \\
Sphere volume (ml) & 0.3 & 0.7 & 1.2 & 1.4 \\
Activity (MBq) & 0.74 & 1.85 & 2.96 & 3.33 \\
\hline
\end{tabular}

FBP method with different filters (Hamming, Hann and Ramp, varying cut-off frequencies) was performed from the raw SPECT data. Regions of interest (ROI) were selected from CT images to obtain the mean and maximum counts per pixel. All measurements were performed in the central section of the lesion (spheres), subtracted from the phantom's body concentration value (background) as show in Figure 3.

Figure 3: Relative quantification in the phantom, Hamming filter, cut-off 0.5: a) BG; b) spheres.
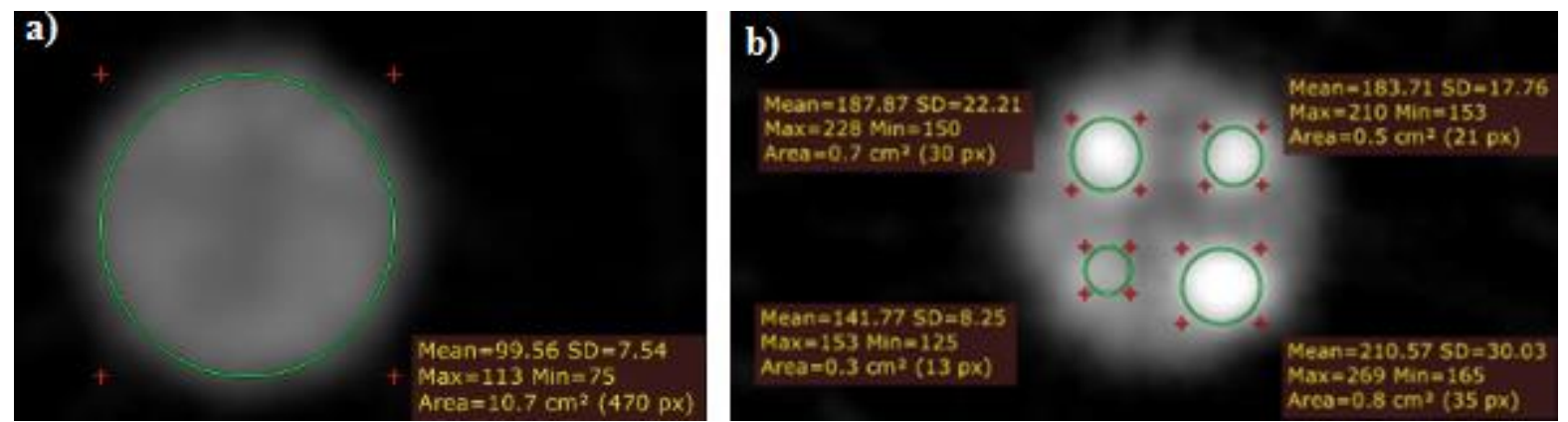
Comparative analyses between different cut-off frequencies and filters were then performed to obtain the reconstruction method that could provide the highest resolution to be used for the dosimetry study in mice pre-clinical study.

\section{RESULTS AND DISCUSSION}

The average relative score curves were studied in function of the lesion diameters for Hamming, Hann and Ramp filters analysing the better cut-off, as exemplified in Figure 4 for Hamming filter. Hann and Ramp filters presented the same pattern as Hamming filter.

Figure 4: Average relative quantification, Hamming filter according to cut-off frequencies

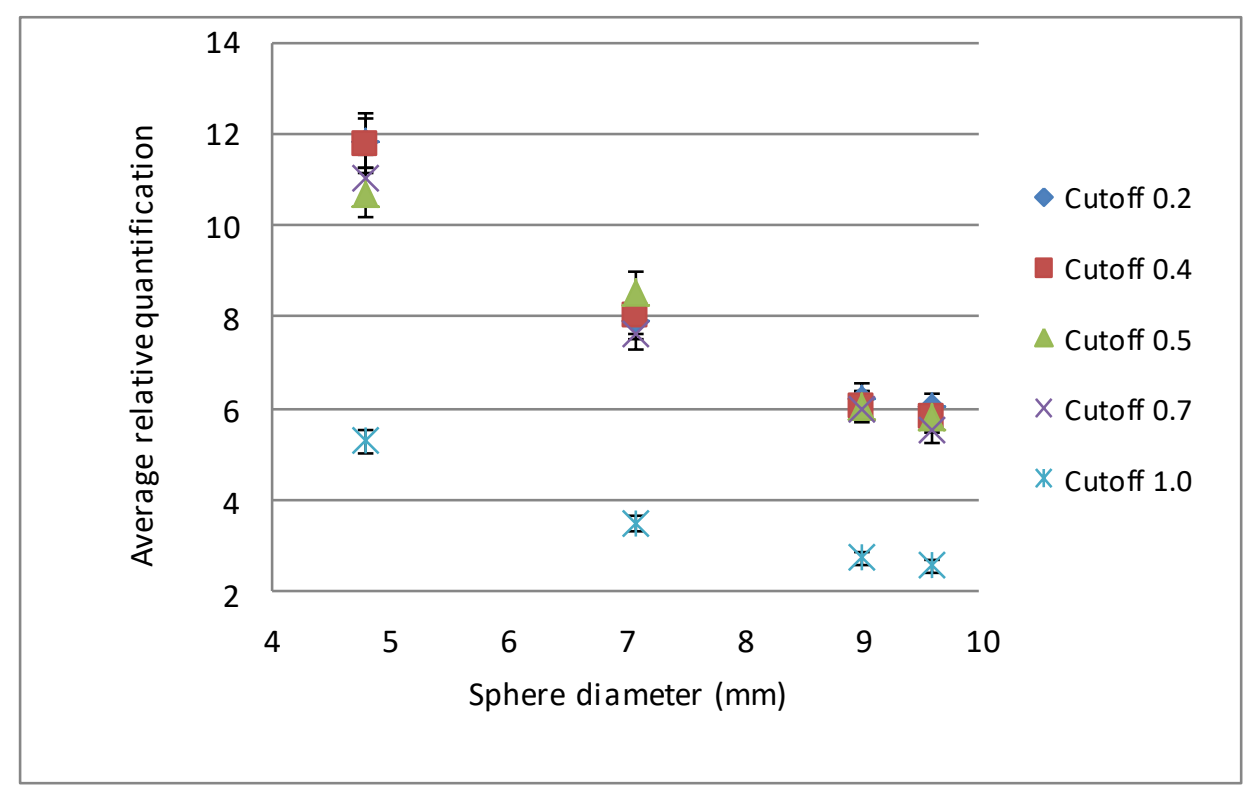

Subsequent analyses were performed with this cut-off frequency of 0.5 since it was the one with the least degraded quantification (Figure 5). 
Figure 5: Average relative quantification per filter for cut-off 0.5

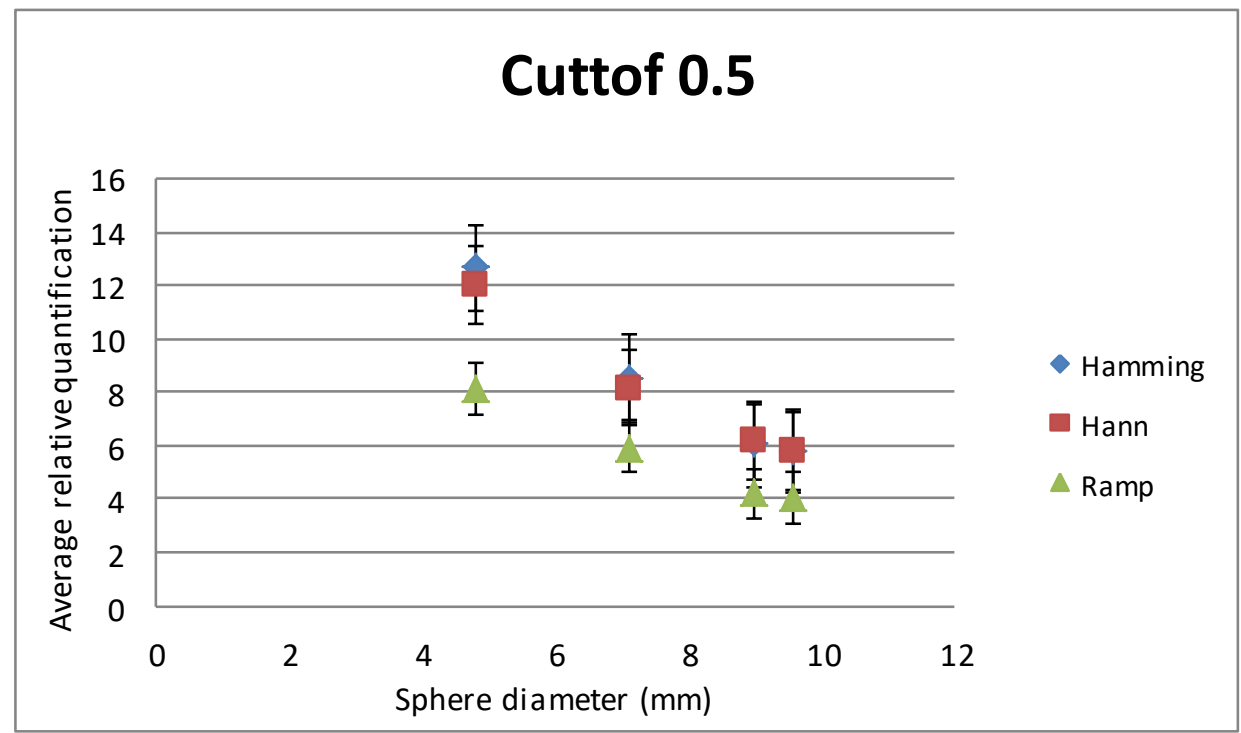

Figure 5 shows that for small diameter lesions, the Hamming filter presented better relative quantification, meaning better contrast.

Spatial resolution of the reconstructed image was determined by the inflection of the average relative quantification curve for each filter, indicating the smaller diameter where the counting remains constant (Figures 6 to 8).

Figure 6: Average relative quantification for Hamming filter cut-off 0.5

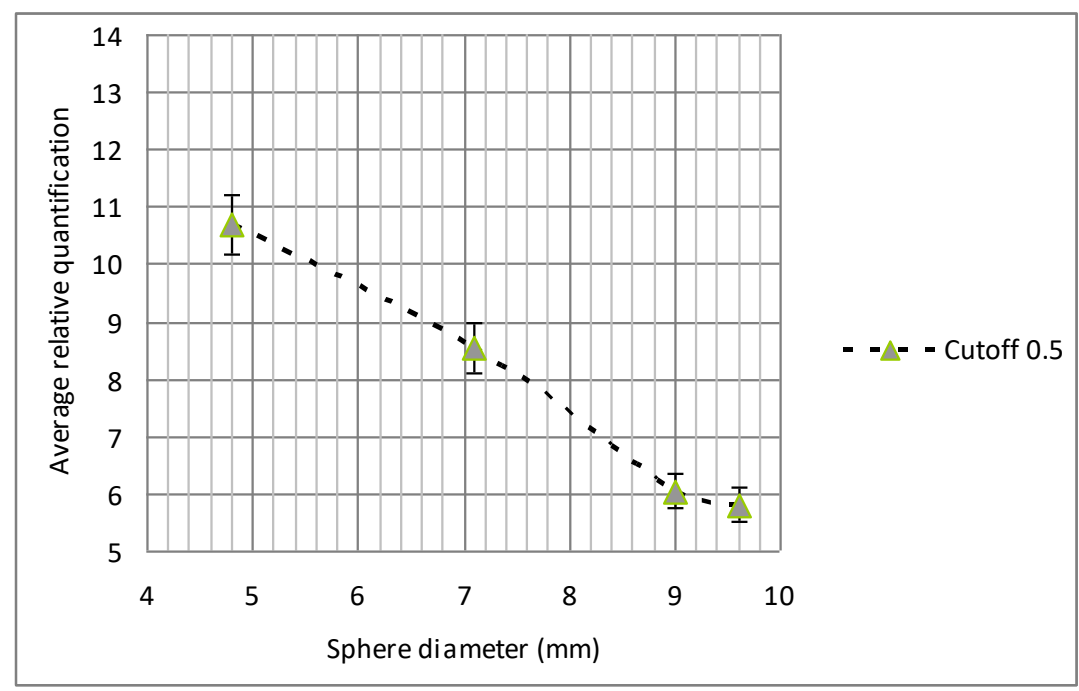


Figure 7: Average relative quantification for Hann filter

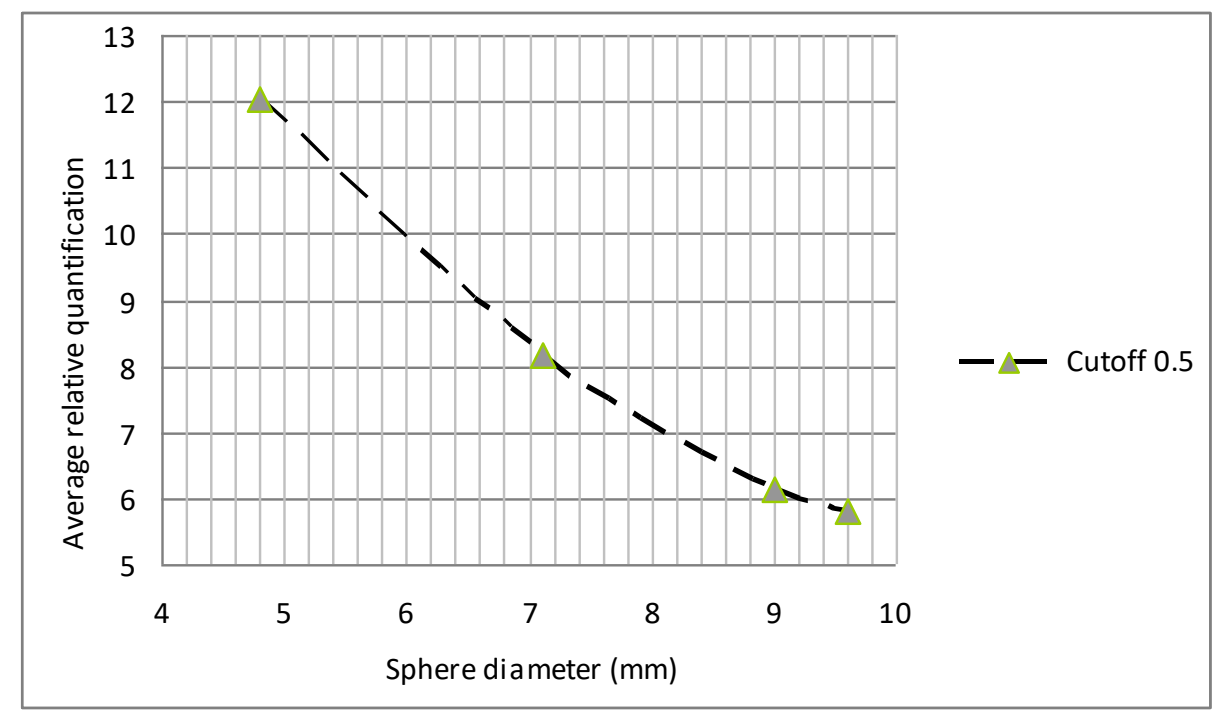

Figure 8: Average relative quantification for Ramp filter, cut-off 0.5

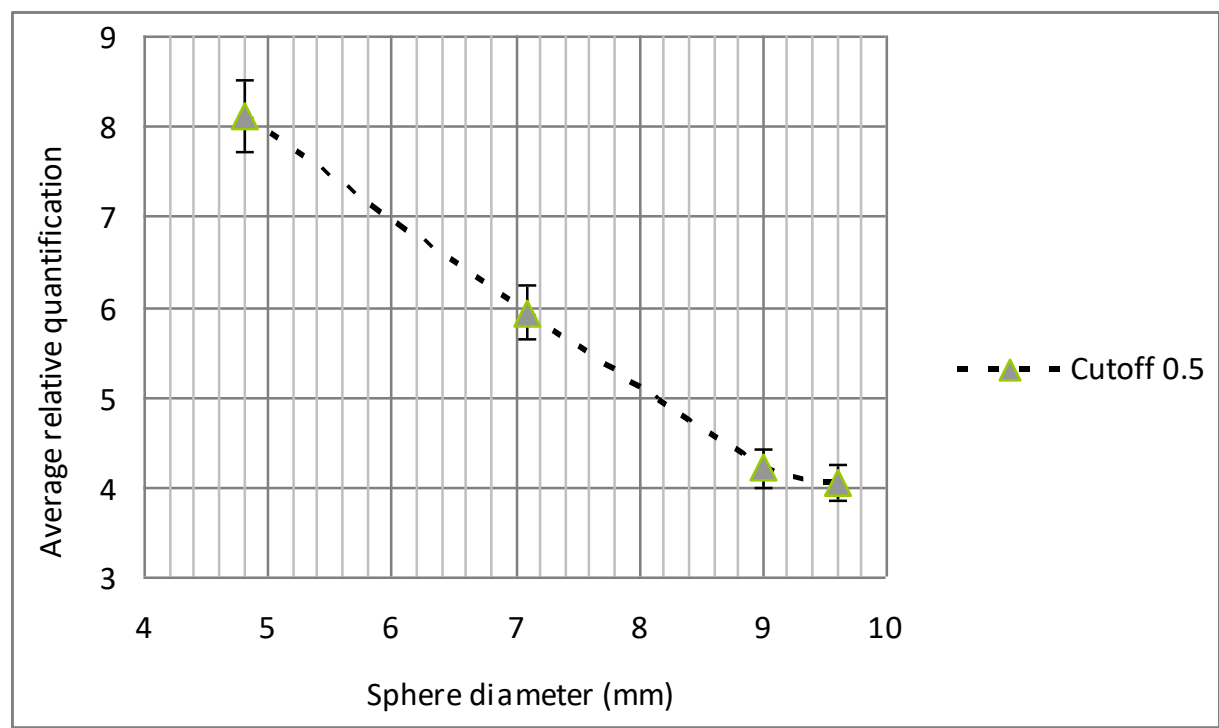

Table 2 describes the image spatial resolution obtained for each tested filters, considering the inflexion point of the curves. 
Soriano, et. Al. • Braz. J. Rad. Sci. • 2018

Table 2: Spatial resolution of the reconstructed image for each filter, considering cut-off 0.5

\begin{tabular}{cccc}
\hline Filter & Hamming & Hann & Ramp \\
$\begin{array}{c}\text { Image Spatial Resolution } \\
(\mathbf{m m})\end{array}$ & 9.2 & 9.2 & 9.0 \\
\hline
\end{tabular}

\section{CONCLUSION}

Ramp filter lead to the best image spatial resolution - $9.0 \mathrm{~mm}$ - of all performed measures, for both average and maximum relative score. However, visual image analysis showed low quality by introducing errors in ROI definition due to edge effects in the lesions. For Hamming and Hann filters, maximum score for the best resolution was $9.2 \mathrm{~mm}$ with 0.5 as cut-off. The reconstructed image resolution between 9.3 to $9.4 \mathrm{~mm}$ is less than the amount provided by the manufacturer manual, $1.6 \mathrm{~mm}$.

Thus, the local protocol for mice can be optimized by FBP reconstruction method and Hamming filter with cut-off of 0.5 , yielding a reconstructed image resolution from 9.3 to $9.4 \mathrm{~mm}$. This value indicates that technetium-uptake region diameters below $9.3 \mathrm{~mm}$ are not quantifiable and that the partial volume effect quantification for lesions smaller than $9.0 \mathrm{~mm}$ must be corrected to perform dosimetry studies.

\section{ACKNOWLEDGMENT}

The authors would like to thank the financial support from CNEN and mechanic technicians from IRD for phantom construction. 


\section{REFERENCES}

MARTIC- KEHL, I. MSCHIBLI, R., SCHUBIGER, A. P. Can animal data predict human outocme? Problems and pitfalls of translational animal research. Eur J Med Mol Imaging,v. 39, p. 1492-1496, 2012.

MEIKLE R S, KENCH P, KASSIOU M, BANATI BR. Small animal SPECT and its place in the matrix of molecular imaging technologies. Phys Med Biol, v. 50, p. R45-R61, 2005.

POWSNER R. A, POWSNER E. R. Essential Nuclear Medicine Physics, Blackwell Publisher, Boston \& United States of American, 2006. 